\title{
Гидродинамика вязкой двумерной электронной жидкости в магнитном поле
}

\author{
Алексеев П.С. \\ ФТИ им. А.Ф. Иоффе, 194021, С.-Петербург, ул. Политехничекская, 26
}

DOI 10.34077/Semicond2019-194

В проводниках с малой плотностью дефектов межэлектронные столкновения могут приводить при низких температурах к формированию вязкой электронной жидкости. Недавно были обнаружены яркие свидетельства наличия такой жидкости в однослойном графене, в вейлевских полуметаллах, в высокоподвижных квантовых ямах на основе GaAs (см. ссылки в [1]-[4]). Одним из этих свидетельств является эффект гигантского отрицательного магнетосопротивления. Действительно, явление вязкости представляет собой диффузный перенос среднего импульса частиц жидкости за счет их свободного движения в промежутках между столкновениями. Для случая электронной жидкости магнитное поле искривляет траектории частиц и сильно сокращает эффективную длину переноса импульса. В связи с этим вязкость электронной жидкости и пропорциональное ей гидродинамическое сопротивление падают с магнитным полем. Расчёты показывают, что такой механизм хорошо объясняет зависящую от температуры часть гигантского отрицательного магнетосопротивления [1].

В недавних экспериментальных работах сообщалось, что в GaAs квантовых ямах высокого качества часто присутствуют макроскопические овальные дефекты. Неоднородное распределения этих дефектов по образцу может приводить к сосуществованию в одном образце областей двух следующих типов [2]. В областях, где дефекты почти отсутствуют, доминируют межэлектронные столкновения и реализуется описанный выше гидродинамический режим транспорта. В областях, где концентрация дефектов высока, электроны сталкиваются чаще всего с границами макроскопических дефектов и поэтому реализуется баллистический режим транспорта. В связи с этим был теоретически изучен магнетотранспорт взаимодействующих двумерных электронов в баллистическом режиме [2]. Обнаружено, что влияние слабого магнитного поля на траектории частиц, сталкивающихся только с краями баллистической области, приводит к температурно-независимому отрицательному магнетосопротивлению. Оценки величины этого эффекта для высокоподвижных GaAs квантовых ям показывают, что такой механизм, по-видимому, ответственен за не зависящую от температуры часть гигантского отрицательного магнетосопротивления, наблюдаемого на этих ямах [2].

В фотосопротивлении высокоподвижных GaAs квантовых ям в магнитном поле был обнаружен гигантский пик на частоте излучения, близкой к удвоенной циклотронной частоте электронов. Для объяснения этого эффекта была построена теория высокочастотного магнетотранспорта вязкой двумерной электронной жидкости [3]. Показано, что коэффициенты вязкости электронов имеют резонанс на частоте излучения, равной удвоенной циклотронной частоте. Физическая природа этого резонанса состоит во вращении тензора вязких напряжений заряженной жидкости в магнитном поле с удвоенной циклотронной частотой. В достаточно широких образцах резонанс может проявляться в затухании магнетоплазмонов [3], а в достаточно узких образцах - в возбуждении волн поперечного нулевого «магнетозвука» [4]. Были рассчитаны закон дисперсии и коэффициент затухания такого звука для случая сильно вязкой электронной жидкости [4]. На основе полученных теоретических результатов даны свидетельства в пользу того, что наблюдаемый в фотосопротивлении гигантский пик представляет собой проявление предсказанного «вязкостного резонанса».

[1] P.S. Alekseev, Phys. Rev. Lett., 117, 166601 (2016).

[2] P.S. Alekseev and M.A. Semina, Phys. Rev. B, 98, 165412 (2018); arXiv:1903.07925 (2019).

[3] P.S. Alekseev, Phys. Rev. B, 98, 165440 (2018).

[4] P.S. Alekseev and A. P. Alekseeva, arXiv:1810.10241 (2018). 